\title{
An Application of Virtual MIMO Technology in WSN Routing Protocol Algorithms
}

\author{
Ying Liang*, Yongxin Feng \\ School of Information Science and Engineering, Shenyang Ligong University, Shenyang, China \\ *Corresponding author’s Email: liangying.slg@163.com
}

\begin{abstract}
MIMO (Multiple-Input Multiple-Out-put) system is a core technology used in 802.11n which used multiple antennas to suppress fading. In the field of WSN network, routing protocols must be designed to minimize energy consumption for the nodes' energy supply is constrained. Using MIMO technique in WSN could make use of the energy accumulation in the receiving nodes to increase the receiver signal intensity, thus reducing bit error rate and improve the reliability of data communication. As the MIMO-based WSN could supply higher data transfer rate for the same transmitting power and bit error rate, it also efficiently reduces the time of the node sending data and improves communication performance of the whole network. In this paper, based on dynamic collaborative virtual antenna MIMO technology, a new energy efficient routing protocol is proposed. The simulation results indicate that our protocol can obviously reduce the energy consumption in the specific application environment.
\end{abstract}

Keywords: MIMO; WSN; routing protocol; virtual antenna

\section{Introduction}

\subsection{Overview of wireless sensor networks}

Wireless Sensor Networks (WSNs) are composed of a large number of battery-powered sensor nodes that have the ability to sense the physical environment, compute the obtained information and communicate using the radio interfaces. Because sensor nodes are generally deployed on a large and wild area, they are powered by embedded battery. As it is difficult to change or recharge the battery, energy-efficient mechanism for wireless communication on each sensor node is so crucial for wireless sensor networks. Generally, WSNs have the following characteristics:

\section{(1) Limited hardware resources}

Due to the price, volume and power restriction of node, in addition to limited energy capacity, sensor nodes have also limited processing and storage capacities.

(2) Huge number of nodes
Sensor node deployment in WSNs is application dependent and can be scattered randomly in an intended area or dropped massively over an inaccessible or hostile region. This request the node must have very strong fault tolerance and survivable.

(3) Self-organization

The network organization does not depend on any fixed network infrastructure. The nodes, after having been deployed, coordinate their respective actions by a set of rules, which can quickly and automatically to form an independent network.

(4) Dynamic topology

In WSN, some nodes could run out of the network for failing or being blocked due to lack of power, or have physical damage or environmental interference. New nodes may be added for the growing network size. Moreover, the mobility of sensor nodes is sometimes necessary in many applications. Therefore network topology changes frequently.

The purpose of the wireless sensor network is to 
collect information timely and reliably. In order to achieve this goal, protocols about the network transmission and topology control must be designed based on the characteristics of the network itself. Since the premise of the WSNs working well is that the sensor nodes have sufficient energy, the design of network protocols must take into consideration the energy consumption factor and need to trade-off between improving network performance and reducing energy consumption. Energy efficiency is not only the key to extend the network lifetime, but also an important foundation to ensure the network is available and reliable, which meeting a certain quality of service.

\subsection{MIMO technology overview}

The origin of MIMO technology has been a long time, back in 1908, Marconi has proposed it to overcome channel fading. In the 70's, some people have suggested to using MIMO technology for wireless mobile communications systems, which is completed by the AT \& T Bell Laboratories scholars in the 90's. In 1995, Teladar theoretically derived from the MIMO capacity under the fading condition. In 1996, Foshini have proposed a new MIMO processing algorithm DBLAST (Diagonal Bell Laboratories Layered Space Time). In 1998, a space-time code for MIMO was discussed by Tarokh. Using V-BLAST (Vertical-BLAST) algorithm, Wolinansky et al have established a MIMO experimental system in 1998 and achieved over 20bps/$\mathrm{HZ}$ frequency spectrum utilization. The above works attract much attention of scholars from various countries and make the MIMO Technology has been developing rapidly [1].

\subsection{The advantages of MIMO technology}

Energy consumption module of sensor node include the sensor module, processor module and wireless communication module. With advances in integrated circuit technology, the processor and sensor module is more and more low-power, and thus, wireless communication module becomes the largest energy-consuming part in a sensor node.

In wireless communications, the relationship between energy consumption and communication distances can be expressed as:

$$
E=k D^{n}
$$

where $n$ is the path loss factor, and the range is $2<$ $n<4$. The value of $n$ depends on many factors such as the deployment environment of sensor nodes, quality of the antenna and so on. The parameter $k$ is a constant, $D$ is the communication distance. According to the formula (1), with the communication distance increases, the energy consumption of wireless communication will increase dramatically. Therefore, in the current wireless sensor networks based on SISO (single input and single output), many protocols use reducing the single-hop communication distance to save transmission energy of nodes. When we use MIMO technique in WSN, it could make use of the energy accumulation in the receiving nodes to increase the receiver signal intensity, thus reduce bit error rate and improve the reliability of data communication. That is, for the same transmitting power and bit error rate, the communication distance in MIMO-based WSN is much larger than that in SISO-based WSN, which efficiently reduces the time of the node sending data and improves communication performance of the whole network.

\section{Related Research and Question Statement}

The research of using MIMO technology in wireless sensor networks first began in 2004 and currently there are some scholars have started working at some of the challenging issues. Literature [2] analyzed the extra energy cost of the communication among cooperation nodes and achieved the total energy consumption in cooperative MIMO-based wireless sensor network. The experimental results show that, even in considering the additional traffic load, collaborative MIMO communications mode still save more energy than SISO communications mode. Literature [3] analyzed the performance of MIMO-based wireless sensor network in the case of the same channel interference. Literature [4] analyzed the diversity gain and multiplexing gain provided by MIMO and proposed the method of using different gains methods in MIMO according to different application requirements.

Some researchers dedicated to study the challenging issues for WSNs using collaborative MIMO, such as how to select collaboration nodes, synchronization, routing and so on. Literature [5] proposed a cooperative node selection algorithm. Each node selects the node from its neighbors as its cooperation node which has the smaller ratio of its transmission energy consumption to its residual energy. Taking into account the similarity of characteristics of collaborative MIMO and clustering WSN, literature [6] proposed using cooperative MIMO techniques in cluster-based wireless sensor networks, which select collaboration nodes from cluster-heads to form collaborative MIMO. 
In order to reduce the total energy consumption in the network, literature [7] proposed a method of optimizing the transmission rate and the size of cluster at the same time. However, the algorithms proposed based on the same assumption, that is, nodes are densely distributed in the network to ensure cluster-heads find enough cooperative nodes. In practice, with the running of network, some nodes could run out of the network for failing or being blocked due to lack of power, or have physical damage or environmental interference. When the network deployment becomes sparser but the amount of information to be transferred is huge, the collaboration nodes could consume more energy than the normal nodes, which aggravates the energy imbalance in the network. Though some protocols use the method of shortening the period of clustering to solve the problem, frequent clustering could waste more energy. In view of the above insufficiency, the paper proposes a dynamic collaborative virtual MIMObased routing protocol (DCVM).

\section{DCVM Routing Protocol}

The basic idea is that it includes the node's residual energy additional information when sending a package, then according to energy left information, the MIMO systems is formed by selecting a certain percentage of nodes as virtual antennas dynamically, namely, DCVM routing protocol.

\subsection{System model}

LEACH algorithm is a typical topology adaptive clustering algorithm. Its implementation is periodic, each round divides into the clustering establishment stage and the stable data communication stage. During the clustering stage, the neighbor node dynamic forms the cluster, the cluster head selected according to certain principles. During the data communication stage, nodes send date to the cluster head, then, cluster head fuse these data and send it to the sink node, LEACH clustering algorithm achieve a good energy saving effective, obtained the widespread attention. This article draws on the idea of LEACH algorithm.

This network model shown in Figure 1, the network is a sparse deploying; Nodes are randomly deployed in a square monitor area for collect data, in above network model, according to the role it plays, nodes can be divided into different three kinds: head node, coordination antenna node and normal node. In this network model, the gathered data is fused by head node, after fusing, the date will be sent to base station by antenna nodes with MIMO-based technology. The vir-

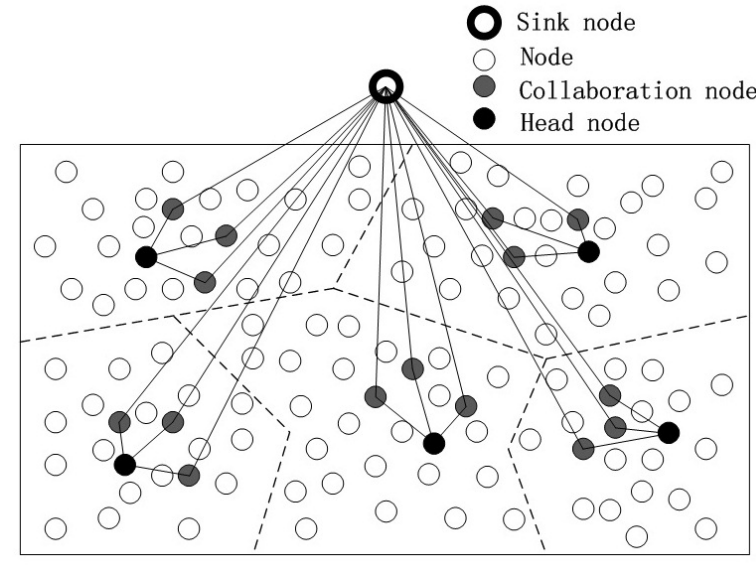

Figure 1 Network Model

tual antenna node is dynamic selects from its neighbor node by cluster head node under certain limiting conditions. When head node communicate with base station, the antenna node and head node dynamic collaborative send package to base station with MIMO patters, by reducing the send error rate and convert to idle quickly, this can save sending energy of networks. In addition to the head node and the antenna node, the remaining nodes shall be normal nodes. Each normal nodes belonging to a cluster, and normal node direct communication with the head node.

Similar to LEACH, DCVM also based on the periodic operation, in each round, first select the cluster head, in the data collection phase, according to the additional residual energy information to form antenna node dynamically, then processing data transmission. When data transmission, firstly, normal nodes send data to head node with the way of SISO. When the cluster head receives and fusion the data send from normal node, then send the data to base station with the way of MIMO which is coded with STBC spacetime code algorithm.

In this network model, assumption that Energy and capacity of the base station is not limits and the base station is of multiple antennas, base station receive space-time coding data by multi-antenna way, and get the original data by space-time decoding. In order to more clearly describe and analysis the energy consumption of network protocols, make the following assumptions:

(1) In the cluster, the distances of normal nodes communication between clusters head is shorter, communication channel is additive white Gaussian noise channel (AWGN) with the path loss factor of 2 . The communication distance between cluster head node and 
base station is longer. Communication channel is frequency non-selective slow fading channel with the path loss factor of 4.

(2) In order to avoid the system is too complicated, ignore the baseband signal processing modules, including source coding, pulse shaping and so on.

(3) The STBC pace-time coding method is used which the encoding rate is 1 .

(4) Each node can work in four different states, namely, sending, receiving, idle and sleep states.

During node transmission data phase, the node consumes energy is the biggest, in the accepting state, the node only receives the data, consumes energy is small, In the idle phase, nodes monitor the use of wireless channel and check whether there is data sending to it, the energy consumption is smaller. In the sleep state, nodes turn off communication module, and then the energy consumption is smallest.

\subsection{Clustering algorithm description}

In the wireless sensor network, each node has a globally unique ID number; the ID number is MAC address of the hardware node. DCVM protocol is divided into four stages, namely, clustering, selection of the cluster head node, dynamic selection of the virtual antennas and data transmission. They are listed as follows:

\subsubsection{Clustering stage}

The selection of Cluster head is similar to the LEACH protocol, in each round, according to its own generated random number and some certain rules to run for cluster head. Constraint rules as follows:

$$
P(i)=\left\{\begin{array}{cll}
\frac{n}{N-n *\left(c \bmod \frac{N}{n}\right)} & \text { if } & \text { flag }(i)=1 \\
0 & \text { if } & \text { flag }(i)=0
\end{array}\right\}
$$

where $N$ is the total number of nodes in the network, $n$ is the number of total clusters. If the node has been cluster head in the last round, then $\operatorname{flag}(i)=0$, otherwise the $\operatorname{flag}(i)=1$, only the residual energy is greater than the threshold of the residual energy, the node has a chance to become a cluster head. This value decided by energy consumption in one round, this stage is the same with the LEACH protocol.

Selection cluster head algorithm as follows:

(1) When a node receives the trigger information from the base station, each node generates a random time $\operatorname{rand}(t)$, which has a ceiling limit value, in network start or running period, the limit value is send from base station to each nodes by broadcasts ways. Through the limit value, each cluster node knows the length of cluster head selection time, according to its randomly generated time rand(t) to run for cluster header selection.

(2) After the random time is ready, then turns on own timer, when the timer reaches the random time $\operatorname{rand}(t)$, within this time, if the node not listen to election information from other nodes, indicates that no node becomes cluster head. Then, in the $\operatorname{rand}(t)$ time, the node broadcast cluster head election information by using CSMA/CA channel access method. Symbolizes that this node success becomes a cluster header. The transmit power value of cluster header selection come from base station by broadcast ways. The value of all nodes are the same, the value determines the communication range of the cluster header.

(3) If a node's timer has not reached the time which a random time rand $(t)$ generates by this node, if the node has receive the message of cluster head node election, it shows that there has a node successfully becomes cluster header within the communication range. Then the node to give up running, so ensure that cluster in the range of the communication has only one cluster header, meanwhile, according to the received header election signal strength, the node estimates the distance from cluster header. And the distance and the cluster header ID are stored in the set of cluster header; therefore, the nodes need a collection to storage cluster header, the table includes the cluster head ID and the distance to the cluster header. Until the end of selection of the cluster header, for any cluster header election message, all the similar method adopted to estimate the distance to cluster header. And store the cluster header ID to the collection of cluster header.

(4) All node's timer will be turn off, when the timer reaches the max limit value which generated in cluster header selection stage. Then, each node who failed to run for cluster header, Select their nearest cluster header as its cluster header in which storage in the collection of cluster header. For join the cluster, it sends a request message to cluster header by using CSMA/CA channel. The information include cluster header ID, the node ID and the node's residual energy information, this could as basis of selection collaboration node. Each cluster header receives a request message from normal nodes, according to the signal strength of the message to estimate the distance to its member, then stores these distance information in the member table. This table has recorded each member node's ID and its residual energy information. After the cluster head is selected and the cluster is formed, 
then the network enter working stages.

\subsubsection{Working stages}

In this study, the network environment is Data perceived frequently. Therefore, in working stages, a large number of data package could received. The data packet contains the node's residual energy information $E_{i-l e f t}$. Cluster header could modify member node's residual energy dynamically according to $E_{i-l e f t}$. Meanwhile, a number of nodes will be selected as virtual antennas nodes, these nodes Forms a virtual antenna matrix. The amount of virtual antennas related to the number of antennas of the base station. The cluster header send command packet to its members, this command packets including: node ID, Wake-up command, the antenna assignment information such as commands. The node who receives the antenna command act as a antenna node. In order to decrease the energy consumption for send command package which caused by the cluster header update the list of virtual antenna frequently with changing of the network information, a lock is added to the collection of antenna node, if within the lock period, the collection doesn't update although some nodes has more residual energy. Before data transmission, cluster header using STBC encode the data package according to the antenna nodes in collection. A general round options is $1 / n T$, and $T$ is each round time slot. When working, in pre-allocate time slot, the nodes in each cluster send data package to its cluster header according to the send power which calculators by cluster header, then the node enter the sleep state for saving energy. When the cluster header receive data which sent from its member complete, it could fusion the data according to a certain rules for reducing data redundancy and saving the sending energy. Then, based on the virtual antenna matrix information encode sending data with STBC algorithm and determine which the antenna node is selected, cluster header will broadcast the compressed data to the virtual antenna node. Then the antenna node sends the encoding data to the base station with the way of multi-antenna collaborative.

\section{Energy Analysis Model}

In order to evaluate energy efficiency of DCVM protocol, the network energy consumption model can be descripted as follows:

(1) It is well known that sending and receiving communication energy consumption accounted for the majority of the total energy consumption. Therefore, the analysis of energy consumption, only consider the energy consumption of sending and receiving, ignoring the energy consumption which in idle and sleep state.

(2) As DCVM protocol is periodic working, after the cluster is established, it can repeat data transmission within the round. So most of the energy consumed in data communication, but the energy consumed in cluster establishment is less, it can be ignored.

(3) Due to the range of cluster is not big and the sensing information has a certain correlation, it should be fused, before sending, for reducing the transmission energy consumption.

According to the description about data transmission stage, the network's energy consumption can be divided into three parts: the communicate energy consumption between normal node and cluster header, the communicate energy consumption that clusters header send data to base station with virtual antenna ways.

And each part is made up of the electric circuits consume energy and transmission consumes energy, Detailed calculations as follows:

\subsection{Cluster communication energy consumption}

In sensor networks, $N$ nodes randomly deploying in a square with length $L$, and the entire network is divided into $n$ clusters, each cluster average $N / n$ nodes. as the distance between node and cluster header is closer, assume that the transmission fading factor is 2 , within the cluster, when a node send $\mathrm{k}$ bits data to its cluster header, the average energy consumption can be expressed as:

$$
E_{\text {Total }}=k E_{r}+k E_{s}+\alpha K E\left[d^{2}\right]
$$

where $E_{s}$ and $E_{r}$ respectively, per bit send/receive consume energy between node and cluster header, $\alpha$ is the parameter related to bit error rate. $E\left[d^{2}\right]$ is the mathematical expectation which the square of the distance between node and cluster header, can be expressed as:

$$
\begin{aligned}
E\left[d_{\text {ch_ch }}^{2}\right] & =\iint\left(x^{2}+y^{2}\right) \rho(x, y) d x d y \\
& =\iint r^{2} \rho(r, \theta) d r d \theta
\end{aligned}
$$

where $\rho(x, y)$ is distribution probability that any position of a node in the cluster, in the entire cluster, assumption that node's distributed probability is the same. Then $\rho(r, \theta)$ independence in $r$ and $\theta . \rho(r, \theta)=$ $\frac{1}{\left(A^{2} / n\right)}$. Assuming that each cluster is a circular area with a radius equal to $R$, it has $R=\frac{A}{\sqrt{\pi n}}$, when each 
node send $k$ bit data to cluster header, each communication consumption energy can be expressed as:

$$
\begin{aligned}
E_{\text {cluster_cn_ch }} & =\left(\frac{N}{n}-1\right) E_{\text {ch_ch }} \\
& =\left(\frac{N}{n}-1\right)\left(K E_{c t}+K l_{\varepsilon f s} \frac{A^{2}}{2 \pi n}+k E_{c r}\right)
\end{aligned}
$$

\subsection{Virtual MIMO energy consumption}

After completion of space-time coding, the collaboration nodes, will send a message to base station in MIMO way. If has the $N_{t}$ node forms collaboration type transmission antenna, Base station equipped with $N_{r}$ receive antennas. it forms the MIMO communication mode. Where, the path loss factor of the communication between cluster header and base station is 4 . And because the base station without energy restriction, the base station's power consumption is not calculated. According to the above, the energy consumption which virtual node sends data to base station by MIMO way is:

$$
E_{c h \_b s}=\frac{N}{n} k r N_{c}\left(E_{c t}+l_{\varepsilon m p} E\left[d_{c h \_b s}^{4}\right]\right)
$$

which $l_{\varepsilon m p}$ is the antenna parameters, this parameter has related with error rate, antenna number $N_{t}$ and base station's antenna number $N_{r} . E\left[d_{c h b s}^{4}\right]$ is the 4 powers mathematical expectation which the distance between cluster header and base station. As the base station is located in the center of the network, assuming the base station height is $H$, then $E\left[d_{c h \_b s}^{4}\right]$ can be expressed as:

$$
E\left[d_{c h \_b s}^{4}\right]=\iint\left(x^{2}+y^{2}+H^{2}\right)^{2} \rho(x, y) d x d y
$$

$\rho(x, y)$ is the distribution probability of cluster header.

$$
E\left[d_{c h \_b s}^{4}\right]=0.04 A^{2}+0.33 A^{2} H^{2}+H^{4}
$$

The cluster energy average consumption is as follows:

$$
E_{\text {cluster }}=E_{\text {cluster_cn_ch }}+E_{\text {ch_sch }}+E_{\text {ch_bs }}
$$

The total energy consumption in a round can be calculated as follows:

$$
\begin{aligned}
E_{\text {total }}= & n E_{\text {cluster }} \\
= & k\left(2 N E_{c}+\frac{N A^{2} l_{\varepsilon f s}}{2 \pi n}-2 n E_{c}-\frac{A^{2} l_{\varepsilon f s}}{2 \pi}\right. \\
& \left.+N E_{f u}+m u M\right)
\end{aligned}
$$

Optimal cluster number $n$ can be calculated as follows:

$$
\begin{aligned}
n_{o p t} & =\frac{A \sqrt{N l_{s}}}{\sqrt{2 \pi} \sqrt{2 E_{c}\left(b N_{c}-1\right)}+T} \\
T= & m l_{\varepsilon f s} d_{\text {max }}^{4}+ \\
& m l_{\varepsilon f s}\left(0.04 A^{2}+0.33 A^{2} H^{2}+H^{4}\right) N_{c}
\end{aligned}
$$

\section{Convergence Rate of DVCM}

The convergence speed is an important indicator of the performance evaluation of algorithmsThe proposed virtual MIMO algorithms, the convergence rate with the nodes in the cluster during the iterations. The convergence rate of DVCM has relations with the iterations of clustering. If the clustering phase requires too much iteration, then the nodes will consume more energy be because of more information want to exchange.

During the clustering phase of DVCM, the iteration termination condition of nodes is $\mathrm{CH}_{\text {prob }}(N)=1$. So as long as known the number of iterations of nodes $N$ when the probability value is 1 , the convergence rate of DVCM can be obtained. From the algorithm description, it's known that After $\mathrm{N}$ iterations, $\mathrm{CH}_{\text {prob }}(\mathrm{N})=2^{\mathrm{N-1}} \mathrm{CH}_{\text {prob }}, \mathrm{CH}_{\text {prob }}$ is initial probability values which calculated according to the formula. For Iteration termination conditions is $\mathrm{CH}_{\text {prob }}$ ( $N)=1$, So $N$ is iterations of the iteration. $N$ is calculated by the following formula

$$
N=\left[\log _{2} \frac{1}{c h_{\text {prob }}}\right]+1
$$

From the above formula, draw a conclusion that the value of $N$ is closely related to the initial selection probability $\mathrm{CH}_{\text {prob }}$ of cluster head. The smaller initial probability value, the greater the number of iterations. Therefore, further solving the maximum limit of $N$, the maximum number of iterations can be obtained.

$$
\begin{aligned}
N & =\lim _{C H_{\text {initial_prob }} \rightarrow P_{\text {min }}}\left[\log _{2} \frac{1}{c h_{\text {prob }}}\right]+1 \\
& =\left[\log _{2} \frac{1}{P_{\text {min }}}\right]+1
\end{aligned}
$$

$P_{\min }$ is a constant in the formula, Therefore, the convergence of DVCM is $\sigma(1)$. When $P_{\text {min }}=0.0005$, the node can be terminated up to 12 times iteration.

\section{Simulation Analysis}

In order to verify the energy efficiency of protocol, we evaluate the performance of DVCM and compare it with the LEACH via simulations. We consider 250 nodes that are uniformly deployed within a square of 200 meter $\times$ 200 meter, and assume that each node has $100 \mathrm{~J}$ of the initial energy. Each node sends 2000 bits of information to its cluster head per round. Taking into account the overhead of processing control information in MIMO-based network, we add 150 bits and 100 bits processing in DVCM and LEACH. The simulation results shown in Figure 2:

Figure 2 shows the total number of nodes that remain alive over the simulation time, where $N_{t}=2$. If the network lifetime is defined as the running time until there are only 180 nodes alive in the network, we can find the network lifetime of DVCM can outperform twice more than LEACH. To have this result is due primarily to the manner of DVCM using MIMO communication in data transmission, which result in the lower BER and higher data 


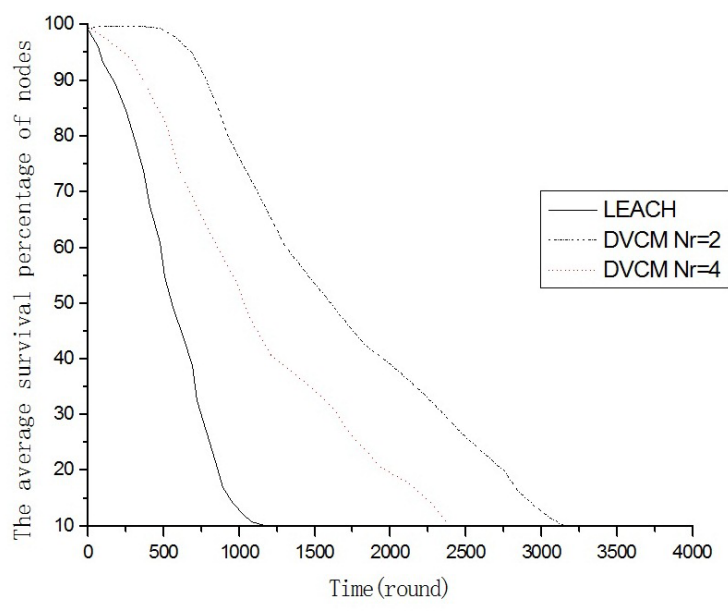

Figure 2 Network nodes running time and the relationship between survival

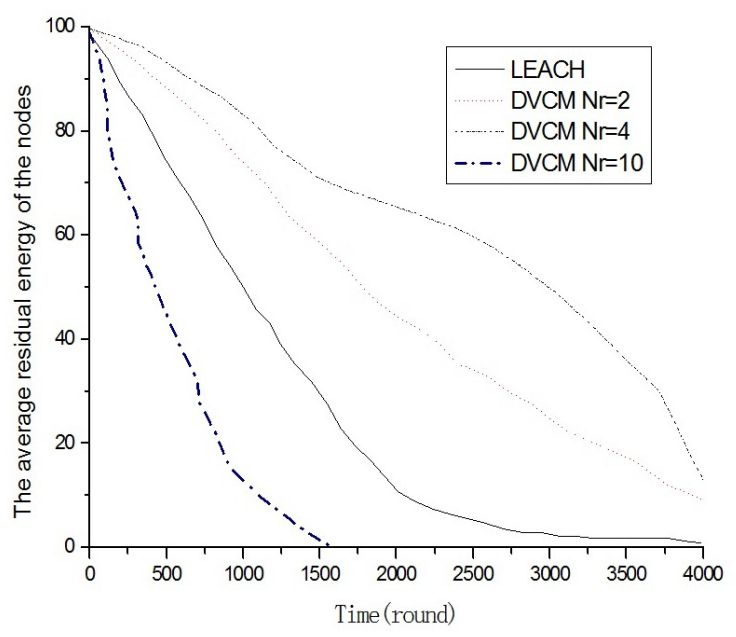

Figure 3 The average residual energy of node and network operation time

throughput within the entire cluster, and thus can save more energy for node rapidly converting from the working state to the sleeping state. In addition, you can also see from Figure 2, the performance of DVCM further improved when the number of virtual antenna node is increased to 4 . This is because more antenna nodes can lead to lower BER and higher data throughput. Therefore, in sparse sensor networks using MIMO technology can significantly improve energy efficiency, thus extending the network lifetime.

From Figure 3, Simulation result can draw the conclusion, as the network operation time increased. DVCM protocol is superior to LEACH protocol. Also show that number of antennas is 4, DVCM protocol with 4 antennas is superior to DVCM protocol with 2 antennas. This is also because of the lower error rate to decrease and data throughput to increase, leading to more energy efficient network. But the simulation also shows that the number of virtual antennas node over $10 \%$ of cluster nodes, The DVCM protocol's performance reduces deeply. Under data frequent sensation environment, this is because of that on the one hand,

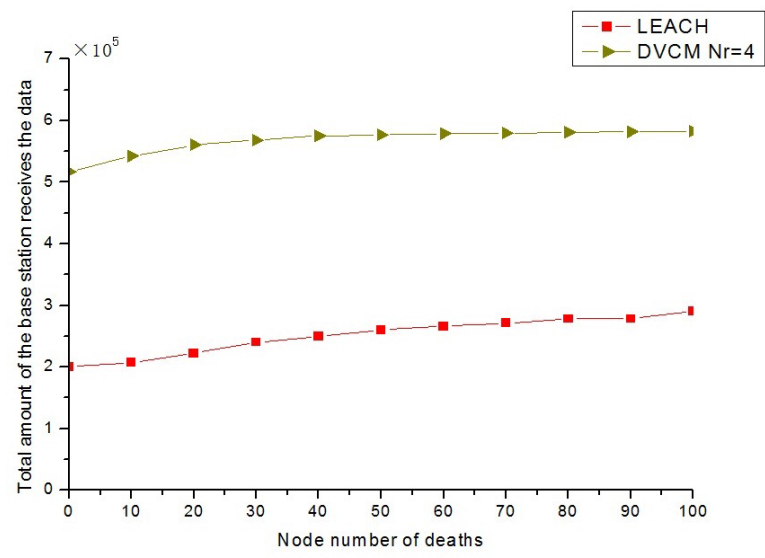

Figure 4 The total number of data received at the BS per given amount of dead nodes

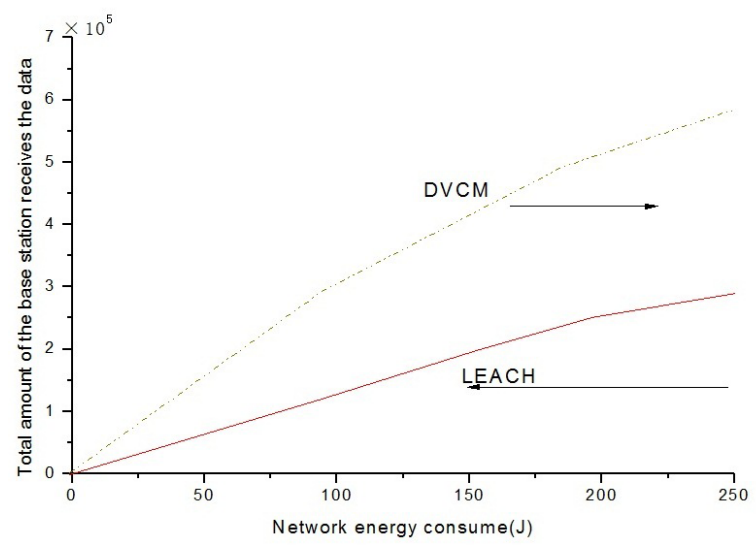

Figure 5 The total number of data received at the BS per given amount of energy consumed

this can increase the complexity of virtual antenna spacetime coding, namely, the computational cost increase, on the other hand, the packet is broken down into more packet data transmission, And because the number of antenna increase, leading nodes send, the receive processing is redundant. So has counter-balanced the advantage which MIMO brings. Generally speaking, the best number of antenna is about $10 \%$ of the nodes in the cluster.

Above simulation results show that under the same number of death nodes, LEACH algorithm for the Amount of receives data of base station which the receives of LEACH algorithm is less than DVCM algorithm. This is because the DVCM using virtual MIMO technology improves the efficiency of data transmission, when transmission the same amount of data, the algorithm of DVCM consumes less energy than consumes of the LEACH algorithm. Therefore, the same numbers of death nodes, the actual network running time of DVCM is longer than the running time of LEACH algorithm. 


\section{Conclusion}

In this paper, DVCM routing protocol is proposed, then the protocol is described in detail, DVCM protocol using hierarchical network topology structure, combine MIMO technology with clustering topology structure, and introducing dynamic virtual antenna mechanism. The problem that cannot find enough nodes as collaborative node, when using MIMO technology in sparse wireless sensor networks, is Solved. Thus succeeds applies the MIMO technology in the sparse network. And, the energy consumption is analyzed; the total consuming energy formula is obtained. By the simulation analysis, DVCM is confirmed which is of the higher performance in the energy conservation aspect.

\section{Acknowledgments}

This work is supported by Doctoral Foundation of Liaoning Province under Grant No.20081027.

\section{References}

[1] C. Shuguang, A. J. Goldsmith and A Bahai, "EnergyEfficiency of MIMO and Cooperative MIMO Techniques in Sensor Network", IEEE Journal on Selected Areas in Communications, vol. 22, Aug. 2004, pp. 1089-1098.

[2] S. K. Jayaweera, "Virtual MIMO-Based Cooperative Communication for Energy-Constrained Wireless Sensor Networks", IEEE Trans. Wireless Commun., Vol. 5, May. 2006, pp. 984-989.

[3] A. M. Abbosh, D. Thiel, "Performance of MIMObased wireless sensor networks with cochannel interference", Proc. International Conference on Intelligent Sensors, Sensor Networks and Information Processing, IEEE Press, Dec., 2005, pp. 115-119.

[4] S. K. Jayaweera, "An Energy-Efficient Virtual MIMO Architecture Based on V-BLAST Processing for Distributed Wireless Sensor Networks", Proc. IEEE International Conference on Sensor and Ad-hoc Communications and Networks(SECON 04), IEEE Press, Oct.,2004, pp. 299-308.

[5] G. N. Bravos, A. G. Kanatas, "Energy efficiency of MIMO-based Sensor Networks with a Cooperative Node Selection Algorithm", Proc. IEEE International Conference on Communications(ICC 07), IEEE Press, Jun. 2007, pp. 3218-3223.

[6] K. R. Xu, Z. Y. Yang, et al, "An End_to_end Optimized Cooperative Transmission Scheme for Virtual MIMO Sensor Networks", Proc. IEEE Singapore International Conference on Communication Systems(ICCS 2006).

[7] S. P. Laxminatayana, V. Krishnamurthy, "Optimal Rate and Number of Clusters in Cluster based Cooperative MIMO Sensor Network", Proc. Signals systems and computers, IEEE Press, 2005, pp. 15561560. 